\title{
Analysis of Models of Oil-by-Water Displacement through the Microstructure
}

\author{
A. M. Meirmanov ${ }^{a}$, G. V. Reshetova ${ }^{b}$, and V. A. Tcheverda ${ }^{c *}$ \\ Presented by Academician M.I. Epov September 7, 2015
}

Received September 7, 2015

\begin{abstract}
Two major arrangements describing the oil-by-water displacement process, i.e., Muskat's model without taking into account the surface tension on the free boundary and the Buckley-Leverett model based on the surface tension, are considered. These arrangements were subject to theoretical and numerical study, which made it possible to uncover their self-contradictoriness. The focus was on construction of a numerical solution at the microlevel using the direct method with pressure correction.
\end{abstract}

DOI: $10.1134 / \mathrm{S} 1028334 \mathrm{X} 1609018 \mathrm{X}$

1. The oil-by-water displacement process is commonly described by two models: Muskat's model without taking into account the surface tension on the boundary [1] and the Buckley-Leverett model taking into account the surface tension on the boundary [2]. They are based on Darcy's law for an absolutely hard rock skeleton. In 1980, L. Tartar in [3] using the averaging technique showed that Darcy's law could be derived from the Stokes equations in a periodically hard skeleton, when the size of the periodicity cell tended to zero. Today, it is possible to apply the approaches focused on the construction and justification of mathematical models of moving fluids in environments with a microstructure, which are based on the following steps:

(1) exact description of moving fluids at the microscopic level (on a pore scale) by the classical equations of continuum mechanics;

(2) mathematically substantiated averaging of the resulting problem and corresponding implementation of the transition from the microlevel to the macrolevel.

Unfortunately, this scheme can be implemented only for single-phase flows [4], so it is particularly important to carry out numerical experiments.

\footnotetext{
${ }^{a}$ Belgorod National Research University, Belgorod, Russia

${ }^{b}$ Institute of Computational Mathematics and Mathematical Geophysics, Siberian Branch, Russian Academy of Sciences, pr. Akad. Lavrentieva 6, Novosibirsk, 630090 Russia

${ }^{c}$ Trofimuk Institute of Petroleum Geology and Geophysics, Siberian Branch, Russian Academy of Sciences, pr. Akad. Koptyuga 3, Novosibirsk, 630090 Russia

*e-mail: Cheverdava@ipgg.sbras.ru
}

2. Let us consider the two-dimensional oil-bywater displacement process in an absolutely hard skeleton. A one-dimensional model of perfect soil is taken as the microstructure: in a rectangle with the unit height there is a system of noncontacting parallel capillaries with a rectangular cross section, whose dimensionless width $\varepsilon$ tends to zero in such a way that the volume porosity remains constant. At the microlevel, the displacement process is modeled in a single capillary:

$$
\Omega=\left\{\mathbf{x}=\left(x_{1}, x_{2}\right):-1<x_{1}<1,-\varepsilon<x_{2}<\varepsilon\right\}
$$

with a moving free surface $\Gamma(t)$ subdividing it into two subregions such as $\Omega^{-}(t)$ and $\Omega^{+}(t)$. Further considerations are performed in dimensionless variables:

$$
\begin{gathered}
\mathbf{x}^{\prime}=L \mathbf{x}, \quad \mathbf{V}^{\prime}=\frac{L}{T} \mathbf{V}, \quad p^{\prime}=p_{0} p, \\
\rho^{\prime}=\rho_{0} \rho, \quad p_{0}=L g \rho_{0},
\end{gathered}
$$

where $L$ and $T$ specify the characteristic length of the capillary and the characteristic time of the process, respectively, $p_{0}$ is the unit pressure (for instance, one atmosphere), and $\rho_{0}$ is the unit density (for instance, water density).

Inside the capillary, the fluid motion is described by the Stokes equations:

$$
\begin{gathered}
\nabla \mathbf{P}^{ \pm}+\rho^{ \pm} \mathbf{g}=0, \quad \mathbf{P}^{ \pm}=\alpha_{\mu}^{ \pm} \mathbf{D}\left(\mathbf{V}^{ \pm}\right)-p^{ \pm} \mathbf{I}, \\
\nabla \cdot \mathbf{V}^{ \pm}=0
\end{gathered}
$$

where $\mathbf{V}^{ \pm}$and $\mathbf{P}^{ \pm}$are the velocity vector and the tension tensor, respectively, to the left and to the right of 


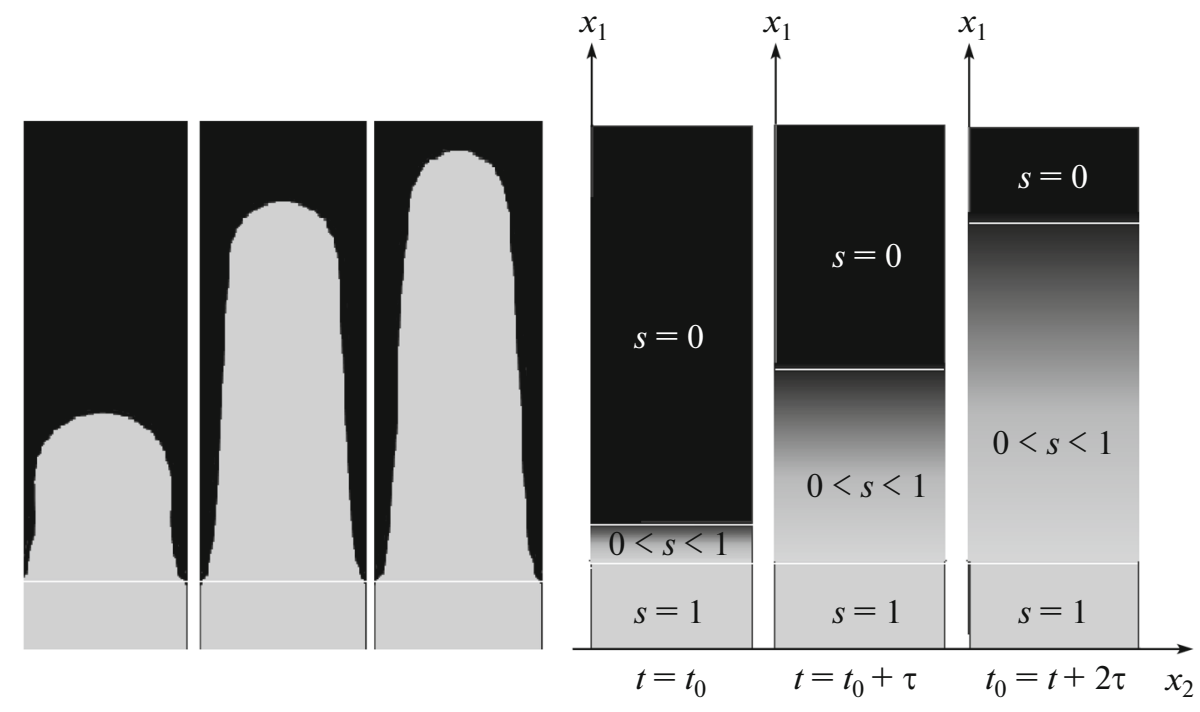

Fig. 1. Numerical modeling of oil-by-water displacement without taking into account surface tension. On the left, change in the border line in one capillary; on the right, flow averaging by periodic replication of the capillary.

the border line $\Gamma(t), \rho^{ \pm}=\frac{\rho^{\prime \pm}}{\rho_{0}}$ and $\alpha_{\mu}^{ \pm}=\frac{\mu^{ \pm}}{T L g \rho_{0}}$ are the dimensionless density and viscosity of fluids, and $\mathbf{D}(\mathbf{V})$ is the strain velocity tensor. Velocities are subject to adhesion conditions throughout the capillary, while pressure is specified in the inlet to which water is supplied and in the outlet from which oil is taken from:

$$
\left.\mathbf{V}^{ \pm}\right|_{x_{2}= \pm \varepsilon}=0,\left.\quad \mathbf{P}^{ \pm} \cdot \mathbf{n}\right|_{x_{1}= \pm 1}=p^{ \pm} \mathbf{n} .
$$

The conditions on the free boundary are specified depending on whether its surface tension is taken into account or not.

Displacement without surface tension. In this case, the consistency of velocities and the normal constituent of the tension tensor is required on the border line $\Gamma(t)$ :

$$
\left(\mathbf{V}^{+}-\mathbf{V}^{-}\right)_{\Gamma(t)}=0,\left(\mathbf{P}^{+} \cdot \mathbf{n}-\mathbf{P}^{-} \cdot \mathbf{n}\right)_{\Gamma(t)}=0,
$$

where $\mathbf{n}$ is the unit normal to the surface $\Gamma(t)$.

Displacement with surface tension. In this case, the border conditions on the free surface are written as follows:

$$
\left(\mathbf{V}^{+}-\mathbf{V}^{-}\right)_{\Gamma(t)}=0, \quad\left(\mathbf{P}^{+} \cdot \mathbf{n}-\mathbf{P}^{-} \cdot \mathbf{n}\right)_{\Gamma(t)}=\frac{\sigma}{R} \mathbf{t},
$$

where $\mathbf{n}$ still specifies the unit normal to the free boundary $\Gamma(t), R$ is its curvature radius, $\mathbf{t}$ is the unit vector of the tangential direction to the border line surface, and $\sigma$ is the surface tension coefficient.

3. Numerical experiments were conducted by direct solution through the pressure correction by tracing the front [5]. The free boundary was constructed by stepwise recalculation with relevant interpolation. The fol- lowing averaging was used to transit from microscale to macroscale:

(1) the displacement task (1)-(3) is solved in the total arrangement under the condition of (4) or (5) for one rectangular capillary with hard walls;

(2) the obtained solution is periodically replicated orthogonally to the capillary axis with simultaneous reduction of its cross section $\varepsilon$ in such a way that the total area occupied by the capillaries remains unchanged, thus providing the specified porosity;

(3) a function with two variables is constructed; it describes the flow in the limit case $(\varepsilon \rightarrow 0)$; due to symmetry of the task, the limit function depends on only one spatial variable, i.e., the capillary length $x_{1}$.

In case of no tension on the boundary, the results of the numerical experiment are given in Fig. 1. Time variations in the form of the boundary between two unmixable fluids in the capillary are shown on the left. The boundary within the capillary is drawn as far as desired due to no surface tension and adhesion condition for the walls. In this case, below the contact (adhesion) points and above the end of the expanded "tongue," there are areas where a volume fraction of water is equal to one and zero, respectively. The mixing zone formed between these areas is characterized by a constant decrease in the volume fraction of water from one to zero. The resulting averaging of the process by the capillary replication with the given porosity is shown on the right. It is seen clearly that the zone formed expands, while the water concentration decreases.

Tension on the free boundary. The calculation results are given in Fig. 2 for the arrangement with account for the surface tension. As before, the time variations in the form of the boundary are shown on 


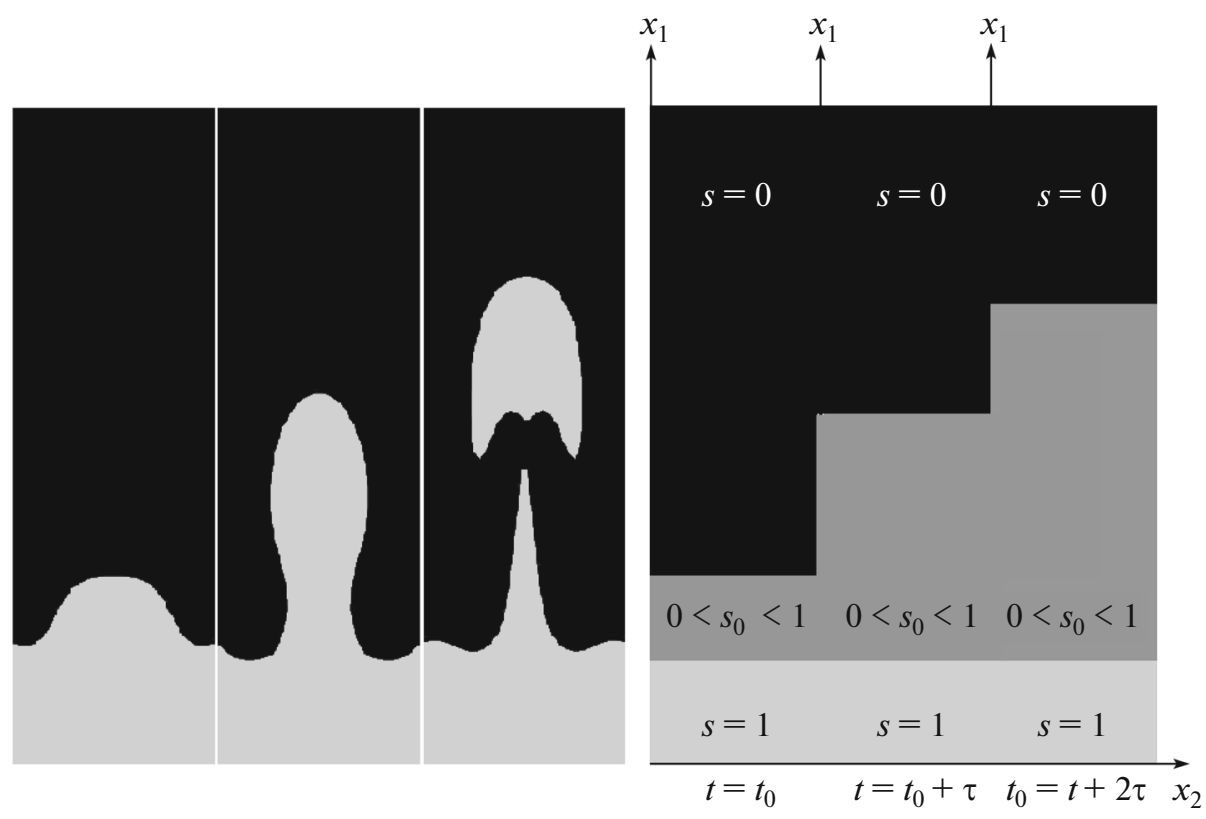

Fig. 2. Numerical modeling of oil-by-water displacement taking into account surface tension. On the left, change in the border line in one capillary; on the right, flow averaging by periodic replication of the capillary.

the left. It is evident that the occurrence of surface tension changes the flow nature considerably. In this case, the conditions for the tension tensor on boundary (5) include the surface tension coefficient and curvature radius. Description of the displacement process follows from the second condition of (5):

(1) under zero pressure drop in the capillary, the curvature radius is infinite, i.e., the boundary is described as a straight line segment;

(2) under increase in pressure, the curvature radius decreases, but while it is more than half of the capillary width, the boundary remains unchanged;

(3) under the critical pressure value,${ }^{1}$ when the curvature radius becomes less than half the thickness of the capillary, drops replacing fluid start forming and "shooting," and their diameter decreases under an increase in pressure.

4. One-dimensional arrangements for Muskat's model and the Buckley-Leverett model allow analytical solutions.

Muskat's model. In the region $\Omega$, two different fluids occupy subregions $\Omega^{ \pm}$separated by the boundary $\Gamma(t)$. Their velocity $\mathbf{V}^{ \pm}$and pressure $p^{ \pm}$are consistent with the system of equations of filtration theory (Darcy's law):

$$
\mathbf{V}^{ \pm}=\frac{k}{\mu^{ \pm}}\left(-\nabla p^{ \pm}+\rho^{ \pm} \mathbf{F}\right), \quad \nabla \cdot \mathbf{V}^{ \pm}=0 .
$$

\footnotetext{
${ }^{1}$ This value depends on the tension coefficient and channel width.
}

On the unknown contact boundary $\Gamma(t)$, the conditions are as follows:

$$
\left[\left(\mathbf{V}^{+}-\mathbf{V}^{-}\right) \cdot \mathbf{n}\right]_{\Gamma(t)}=0, \quad\left(p^{+}-p^{-}\right)_{\Gamma(t)}=0,
$$

where $k$ is the permeability coefficient, $\mu^{ \pm}$and $\rho^{ \pm}$are the fluid viscosity and density, and $\mathbf{F}$ is the given external force. The task is completed by the boundary materiality requirement (the boundary moves along with the flow):

$$
V_{n}=\mathbf{V}^{ \pm} \cdot \mathbf{n}
$$

where $V_{n}$ is the boundary movement velocity, under the initial conditions

$$
\Gamma(0)=\Gamma_{0}
$$

and under the limit conditions

$$
\left.p^{ \pm}\left(x_{1}, x_{2}, t\right)\right|_{x \in S^{ \pm}}=p_{0}^{ \pm},\left.\quad \mathbf{V} \cdot \mathbf{n}\right|_{x \in S^{0}}=0,
$$

where $S^{ \pm}$are surfaces where fluid is pumped and taken, while $S^{0}$ is the surface limiting the fluid flow area.

In the case of one spatial variable, the region $\Omega$ is a segment of the real axis, while the boundary $\Gamma(t)=\{x=X(t)\}$ is specified by one point. The set task is solved explicitly, and the boundary movement $\Gamma(t)=\{x=X(t)\}$ is defined by the following equation:

$$
\frac{d X}{d t}=k \frac{\left(p_{0}^{-}-p_{0}^{+}\right)+\left(\rho^{-}-\rho^{+}\right) X+\left(\rho^{-}+\rho^{+}\right)}{\left(\mu^{-}-\mu^{+}\right) X+\left(\mu^{-}+\mu^{+}\right)} .
$$




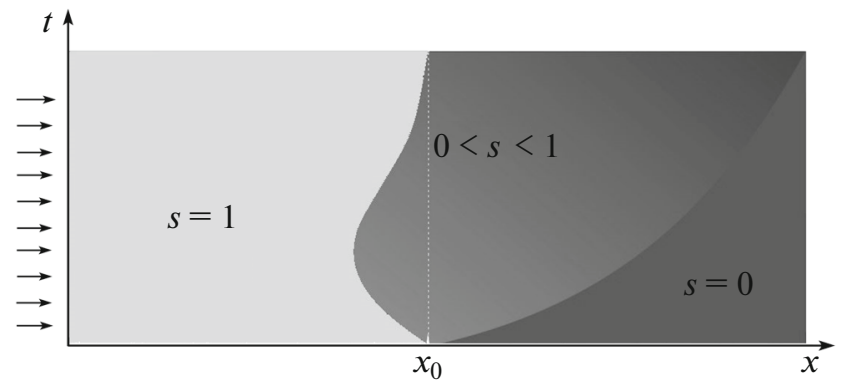

Fig. 3. Automodel solution for the Buckley-Leverett onedimensional model. The artefact that should be noted: flow against the pressure direction at the initial moment of time.

These relations are indicative of the fact that Muskat's model in one dimension describes the so-called "piston" displacement, without the mixing zone. Consequently, it should not be obtained by averaging of the considered idealized model, and therefore, it is incorrect to describe the filtering process even in such a simple environment.

The Buckley-Leverett model. Let us consider again the region $\Omega$, which consists of two nonintersection regions $\Omega^{ \pm}$separated by the boundary $\Gamma(t)$. In each subregion, the velocity, pressure, and concentration $s$ of the fluid pumped from the left from the volume with a "minus" index are related by the following system of equations:

$$
\begin{array}{cc}
\mathbf{V}^{-}=\frac{k^{-}(s)}{\mu^{-}}\left(-\nabla p^{-}+\rho^{-} \mathbf{F}\right), & \frac{\partial s}{\partial t}+\nabla \cdot \mathbf{V}^{-}=0, \\
\mathbf{V}^{+}=\frac{k^{+}(s)}{\mu^{+}}\left(-\nabla p^{+}+\rho^{+} \mathbf{F}\right), & -\frac{\partial s}{\partial t}+\nabla \cdot \mathbf{V}^{+}=0
\end{array}
$$

and the condition relating pressures,

$$
p^{-}-p^{+}=P_{c}(s) \text {. }
$$

Functions $k^{ \pm}(s)$ and $P_{c}(s)$ specifying permeability and capillary pressure, respectively, depending on the concentration $s$, are considered to be predetermined.

The automodel solution for this model demonstrated in Fig. 3 is given in [6]. It differs substantially from the solution obtained by one-dimensional numerical averaging. Moreover, it is characterized by a nonphysical artifact, i.e., flow in the direction opposite to the pressure difference, which is impossible in the one-dimensional case.

5. Numerical one-dimensional averaging of the two-phase flow in a rectangular capillary with hard walls made it possible to determine the characteristic features for two typical arrangements: with surface tension on the free boundary and without it. In the case of no surface tension, the mixing zone is formed and expanded and water concentration in it is constantly reduced from one to zero. In the case of surface tension, two flow conditions are possible: the free boundary is fixed, if the pressure of the supplied fluid is balanced by the surface tension; increase in pressure results in separation of fluid drops supplied from the free boundary and their further distribution along the capillary. Consequently, the expanding mixing zone is formed here as well, but now with a constant water concentration dependent on the number of drops detached from the free boundary. Meanwhile, analytical solutions obtained for the same idealized geometry of the pore space using Muskat's model and the Buckley-Leverett model yield a completely different structure of the flow, thus raising serious doubts as to their physical correctness.

\section{ACKNOWLEDGMENTS}

This work was supported by the Russian Science Foundation, project no. 14-17-00556.

\section{REFERENCES}

1. M. Muskat, Physics, No. 5, 250-264 (1934).

2. S. E. Buckley and M. C. Leverett, Trans. AIME 146, 107-116 (1942).

3. L. Tartar, Lect. Notes Phys. 129, 368-377 (1980).

4. A. Meirmanov, Mathematical Models for Poroelastic Flows (Atlantis Press, Paris, 2013).

5. A. Prosperetti and G. Trygvasson, Computational Methods for Multiphase Flow (Cambridge Univ. Press, Cambridge, 2008).

6. A. Meirmanov and S. Shmarev, Electron. J. Differential Equations 227, 1-13 (2014).

Translated by E. Maslennikova 